\title{
Estação TABA: Uma Infra-estrutura para Implantação do Modelo de Referência para Melhoria de Processo de Software
}

\author{
Ana Regina Rocha, Mariano Montoni, Gleison Santos, Sômulo Mafra, \\ Sávio Figueiredo, Adriano Bessa, Paula Mian \\ COPPE/UFRJ - Universidade Federal do Rio de Janeiro \\ Caixa Postal 68511 - CEP 21945-970 - Rio de Janeiro, Brasil \\ \{darocha, mmontoni, gleison, somulo, savio, bessa, pgmian\}@cos.ufrj.br
}

\begin{abstract}
Recent researches about quality in the software area demonstrate that it is necessary a great effort to improve software processes. This work describes an approach to support the deployment of a Reference Model for Software Process Improvement in Brazil. The preliminary results of the deployment of such model and the functionalities of the TABA Workstation are described in this paper.
\end{abstract}

Resumo. Pesquisas recentes sobre qualidade na área de software demonstram que é necessário um esforço para melhorar processos de software. Este trabalho descreve uma abordagem para apoiar a implantação do Modelo de Referência para melhoria de processo de software Brasileiro. Os resultados iniciais da implantação deste modelo e as funcionalidades da Estação TABA são descritos neste trabalho.

\section{Introdução}

Principalmente no Brasil, existe uma necessidade para melhorar o desempenho de processos de software com o objetivo de aumentar a qualidade dos produtos de software e aumentar as vantagens competitivas de organizações brasileiras tanto no mercado nacional quanto internacional. Desde 1993, com a fundação do PBQP Software (Subcomitê do programa Brasileiro de Qualidade e Produtividade), o Brasil investe em melhoria de Qualidade de Software [Weber et al. 1995] [Weber et al. 2001]. No entanto, um estudo comparativo do MIT (Massachusetts Institute of Technology) [Veloso et al. 2003] concluiu que empresas brasileiras possuem mais interesse na ISO 9000 [ISO 9001, 2000] que outros modelos e padrões especificamente orientados para software.

Esta informação é corroborada por resultados de uma pesquisa do MCT (Ministério de Ciência e Tecnologia do Brasil) [MCT, 2002]. De acordo com esta pesquisa, o número de empresas desenvolvedoras de software no Brasil em 2003 com certificação ISO 9000 era de 214, e o número de empresas com avaliação oficial SWCMM (Capability Maturity Model for Software) era de apenas 30 e nenhuma com avaliação oficial CMMI (Capability Maturity Model Integration) [Chrissis et al. 2003]. Considerando as 30 empresas com avaliação oficial SW-CMM, pode-se verificar que na base da pirâmide há 24 no Nível de Maturidade 2, e 5 empresas no Nível de Maturidade 3. No topo da pirâmide, há apenas uma empresa no Nível de Maturidade 4 e nenhuma no Nível de Maturidade 5. 
Esses dados evidenciam que para melhorar os processos de software no Brasil, dois problemas devem ser resolvidos: (i) com relação ao topo da pirâmide, a questão a ser resolvida é: Como aumentar significativamente o número de empresas brasileiras com avaliação oficial CMMI nos Níveis de maturidade 4 e 5 focando empresas que exportam software e outras grandes empresas?; (ii) com relação à base da pirâmide, existe outra questão que necessita ser respondida: Como melhorar radicalmente os processos de software no Brasil focando em número significativo de pequenas e médias empresas de tal forma que estas empresas possam alcançar Níveis de Maturidade 2 e 3 do CMMI com custos viáveis?

A solução do segundo problema no contexto do projeto de melhoria de processo de software Brasileiro (mps Br) [Weber et al., 2004] foi o desenvolvimento de um Modelo de Referência para melhoria de processo de software brasileiro (MR mps) e um método de avaliação. O MR mps tem sido implantado em diversas empresas brasileiras localizadas no estado do Rio de Janeiro através de um Ambiente de Desenvolvimento de Software (ADS), chamado Estação Taba [Oliveira et al. 2004]. Este ambiente foi configurado e instalado em cada uma dessas empresas com o objetivo de facilitar e acelerar a definição, implantação e melhoria de processos de software. Para avaliar a adequação dos processos implantados e do ADS de apoio, um estudo foi planejado e executado.

A seção 2 apresenta o Modelo de Referência para Melhoria de Processo de Software. A experiência piloto sobre a implantação da abordagem apresentada em empresas de software brasileiras e as funcionalidades principais do ADS de apoio são apresentadas na seção 3. Os resultados práticos da implantação do MR mps em pequenas e médias empresas Brasileiras e os resultados da execução de uma avaliação experimental são apresentados na seção 4. Finalmente, as seções 5 e 6 apresentam algumas lições aprendidas e apontam direções futuras e conclusões do trabalho, respectivamente.

\section{O Projeto mps Br}

O Projeto mps Br tem como objetivo melhorar os processos de software de médias e pequenas empresas brasileiras a custos viáveis. Não é objetivo deste projeto definir algo completamente novo com relação a padrões e modelos de maturidade.

No Brasil, algumas instituições e um número razoável de Agentes SOFTEX têm experiência na formação e gerência de grupos de empresas com o objetivo de melhorar processos de software através de implantação e certificação de padrões 9000 [Weber et al. 1997] e implantar e realizar avaliações oficiais SW-CMM e CMMI. A partir dessas experiências, o Projeto mps $\mathrm{Br}$ foi concebido com o objetivo de tratar duas situações: (i) adaptar e implantar um Modelo de Referência para melhoria de processo de software (MR mps) para empresas individualmente; e (ii) adaptar e implantar o MR mps cooperativamente em pequenas e médias empresas organizadas em grupos para diminuir os custos de implantação através de divisão dos custos totais e facilitar a procura por fontes de apoio financeiro [Weber et al., 2005].

O Projeto mps $\mathrm{Br}$ consiste em 6 fases. O objetivo da primeira fase, concluída em março de 2004, foi organizar o projeto, estabelecer seus objetivos e definir a primeira versão do Modelo de Referência. A segunda fase, concluída em junho de 2004, tinha o 
objetivo de melhorar o Modelo de Referência, iniciar as atividades de treinamento no modelo e executar experiências iniciais na implantação do MR mps em empresas de desenvolvimento de software. Uma dessas experiências foi executada no Rio de Janeiro e está descrita neste trabalho. As demais fases consistem na implantação em paralelo do Modelo de Referência em diferentes partes do país.

O objetivo principal do Projeto mps $\mathrm{Br}$ é criar e disseminar o Modelo de Referência para melhoria de processo de software (MR mps). Não é objetivo do projeto, como mencionado anteriormente, definir algo novo com relação a padrões e modelos de maturidade. A novidade do projeto é a estratégia adotada para sua implantação que considera as características das empresas brasileiras. Além disso, o Modelo possui grandes potenciais de replicabilidade em diferentes regiões do Brasil e em outros países com características similares, por exemplo, países da América Latina.

O ponto inicial, portanto, para a definição do MR mps [Weber et al. 2004] foi a análise das empresas brasileiras, as normas ISO/IEC 12207 [ISO 12207, 2000] e ISO/IEC 15504 [ISO 15504, 2003] e o modelo CMMI [Chrissis et al., 2003]. O padrão de referência para os processos de software do MR mps é a ISO/IEC 12207, isto é, este padrão é o framework para definição dos processos que constituem o MR mps. Da mesma forma que a norma ISO/IEC 12207, o MR mps define processos fundamentais, processos de apoio e um processo de adaptação. Cada empresa interessada em implantar o MR mps deve selecionar os processos pertinentes a partir desse conjunto de acordo com o processo de adaptação. Os resultados esperados com a implantação dos processos MR mps são uma adaptação dos resultados esperados dos processos e atividades da ISO/IEC 12207.

Sete níveis de maturidade foram estabelecidos no MR mps, ordenados do maior para o menor nível de maturidade, são:

- Nível A - Otimização;

- Nível B - Gerenciado Quantitativamente;

- Nível C - Definido;

- Nível D - Largamente Definido;

- Nível E - Parcialmente Definido;

- Nível F - Gerenciado; e

- Nível G - Parcialmente Gerenciado.

Para cada um desses níveis de maturidade, processos foram atribuídos baseado na norma ISO/IEC 12207 e nas áreas de processo dos níveis 2, 3, 4 e 5 da representação em estágios do CMMI (como pode ser visto na Tabela 1). Esta divisão tem uma diferente graduação da representação em estágios do CMMI com o objetivo de permitir uma implantação mais gradual e adequada em pequenas e médias empresas brasileiras. A possibilidade de atribuir níveis de maturidade em empresas considerando mais níveis, além de diminuir o custo e esforço de alcançar um determinado nível de maturidade, também permite uma maior visibilidade dos resultados da melhoria dos processos dentro da empresa e através do país em um espaço de tempo menor se comparado com outros modelos, por exemplo, o CMMI. 
Tabela 1 - Estrutura do MR mps

\begin{tabular}{|l|l|l|}
\hline Nível MR mps & Nível CMMI & \multicolumn{1}{|c|}{ Nome dos Processos } \\
\hline A (mais alto) & 5 & $\begin{array}{l}\text { Inovação e Desdobramento Organizacional, Análise e } \\
\text { Resolução de Causas }\end{array}$ \\
\hline B & 4 & $\begin{array}{l}\text { Desempenho do Processo Organizacional, Gerência } \\
\text { Quantitativa do Projeto }\end{array}$ \\
\hline C & 3 & Análise de Decisão e Resolução, Gerência de Riscos \\
\hline D & 3 & $\begin{array}{l}\text { Desenvolvimento de Requisitos, Solução Técnica, } \\
\text { Integração do Produto, Instalação do Produto, } \\
\text { Liberação do Produto, Verificação, Validação }\end{array}$ \\
\hline E & 3 & $\begin{array}{l}\text { Treinamento, Avaliação e Melhoria do Processo } \\
\text { Organizacional, Definição do Processo Organizacional, } \\
\text { Adaptação do Processo para Gerência de Projeto }\end{array}$ \\
\hline F & 2 & $\begin{array}{l}\text { Medição, Gerência de Configuração, Aquisição, } \\
\text { Garantia da Qualidade }\end{array}$ \\
\hline G (mais baixo) & 2 & \begin{tabular}{l} 
Gerência de Requisitos, Gerência de Projeto \\
\hline
\end{tabular} \\
\hline
\end{tabular}

\section{Experiência Piloto e Ambiente de Apoio}

O MR mps foi implantado pela COPPE/UFRJ em 18 pequenas e médias empresas localizadas no Rio de Janeiro formando dois grupos organizados pela RioSoft (organização não-governamental que integra o Programa Softex - Sociedade para Promoção da Excelência do Software Brasileiro). Estas empresas compartilharam as mesmas atividades de treinamento, que constituíram 44 horas de aulas em tópicos de Engenharia de Software e 20 horas no MR mps e nos processos organizacionais a serem implantados. Três estágios para implantação desses processos foram definidos. Algumas empresas optaram por iniciar seu processo de melhoria seguindo rigorosamente os níveis de maturidade do MR mps e, conseqüentemente, concentrando seus esforços iniciais nas áreas de processo do nível de maturidade G. Outro grupo de empresas decidiu iniciar o trabalho ficando nos níveis de maturidade $\mathrm{F}$ e G, ou seja, estas empresas decidiram tratar todas as áreas de processos equivalentes ao nível 2 de maturidade do CMMI. Uma única empresa decidiu iniciar a partir do nível de maturidade E, pois os seus processos já estavam definidos e implantados. Estas três estratégias são perfeitamente compatíveis com o MR mps e alinhadas aos objetivos do projeto mps Br.

Para apoiar a implantação do modelo, essas empresas contaram com os consultores em processo de software da COPPE/UFRJ e ferramentas CASE integradas em um Ambiente de Desenvolvimento de Software, chamado Es tação Taba [Montoni et al. 2004] [Montoni et al. 2004a] [Montoni et al. 2004b] [Farias et al. 2003].

\subsection{A $\mathcal{E} \operatorname{stação~} \mathcal{T} a b a$ : um Ambiente de Desenvolvimento de Software para Apoiar a Definição, Implantação e Melhoria de Processos}

Ambientes de Desenvolvimento de Software (ADS) têm tido um importante papel para apoiar engenheiros de software na execução de processos de software através da aplicação de procedimentos específicos que combinam ferramentas integradas e técnicas de acordo com paradigmas particulares de software. Além disso, ADSs estão evoluindo 


\section{Simpósio Brasileiro de Qualidade de Software}

para integrar atividades de gerência de conhecimento dentro dos processos de software com o objetivo de apoiar desenvolvedores na produção mais eficiente de produtos de software de melhor qualidade baseado em conhecimento organizacional e experiências passadas [Montoni et al. 2004b].

A Estação $\mathcal{T} a b a$ é um meta-ambiente de desenvolvimento de software criado para apoiar atividades de gerência de projetos, melhoria da qualidade dos produtos de software, e aumento da produtividade, provendo o meio para que engenheiros de software possam controlar o projeto e medir a evolução das atividades baseada em informações coletadas ao longo do desenvolvimento. A Es tação $\mathcal{T} a b a$ também provê a infra-estrutura para o desenvolvimento e integração de ferramentas de apoio à execução de processos de software. Além do mais, esta infra-estrutura mantém um útil repositório contendo informações do projeto de software coletadas ao longo do seu ciclo de vida.

Para apoiar a definição, implantação e melhoria de processos definidos de acordo com o Modelo de Referência apresentado na seção anterior, a Es tação $\mathcal{T} a b a$ apóia a definição de processos padrões da organização e a adaptação desses processos para projetos específicos com o objetivo de aumentar o controle e melhorar a qualidade dos produtos de software. Portanto, a Estação $\mathcal{T}_{a} b a$ não apenas apóia engenheiros de software na execução das atividades dos processos de desenvolvimento de software, mas também provê o meio para executar esses processos de acordo com os processos de desenvolvimento de software da organização.

A Estação Taba evoluiu durante os últimos anos para apoiar atividades de gerência de conhecimento integradas aos processos de software com o objetivo de preservar o conhecimento organizacional e permitir a institucionalização de uma organização de software de aprendizado. Dessa forma, os objetivos principais da Es tação Taba são: (i) apoiar a configuração de ambiente de desenvolvimento de software centrados em processos para diferentes organizações (ADS Configurados); (ii) apoiar a geração automática (instanciação) de ambiente de desenvolvimento de software para projetos específicos (ADS Orientados a Organização); (iii) apoiar o desenvolvimento de software através da utilização de ambientes instanciados; e (iv) apoiar a gerência de conhecimento organizacional relacionada a processos de software.

As ferramentas da $\mathcal{E s}$ taç ão $\mathcal{T} a b a$ oferecem apoio automatizado para:

(i) adaptação dos processos padrões da organização para um projeto específico [Villela et al. 2004];

(ii) definição da estrutura organizacional [Santos et al. 2004];

(iii) aquisição, filtragem, empacotamento e disseminação de conhecimento organizacional [Montoni et al. 2004a];

(iv) planejamento da organização de projetos específicos;

(v) monitoração e controle de tempo, custos, e riscos [Farias et al. 2003], e planejamento de recursos humanos [Santos et al. 2004];

(vi) planejamento e execução de atividades de Gerência de Configuração;

(vii)identificação de requisitos de qualidade de produtos de software; 


\section{Simpósio Brasileiro de Qualidade de Software}

(viii) planejamento de documentação;

(ix) apoio ao planejamento e execução de ações corretivas;

(x) apoio nas atividades de medição e análise baseadas no método GQM (GoalQuestion-Metric) [Basili et al. 1994];

(xi) monitoração de projeto através da geração de relatórios periódicos e medidas;

(xii)controle das atividades executadas durante um projeto específico;

(xiii)gerência de requisitos; e

(xiv) análise post mortem.

Através do ambiente configurado, cada organização pode instanciar um ambiente específico para apoiar a execução do projeto piloto através da ferramenta AdaptPro [Villela et al. 2004]. Através desta ferramenta, o engenheiro de software pode executar as seguintes atividades: (i) caracterizar o projeto; (ii) planejar o processo que irá guiar a execução do projeto através da adpatação de um processo padrão organizacional considerando as características do projeto; e (iii) instanciar um ADS (Ambiente de Desenvolvimento de Software) para apoiar a execução de um processo planejado. A Figura 1 apresenta a tela da ferramenta $\mathcal{A d a p t} \operatorname{Pr} o$.

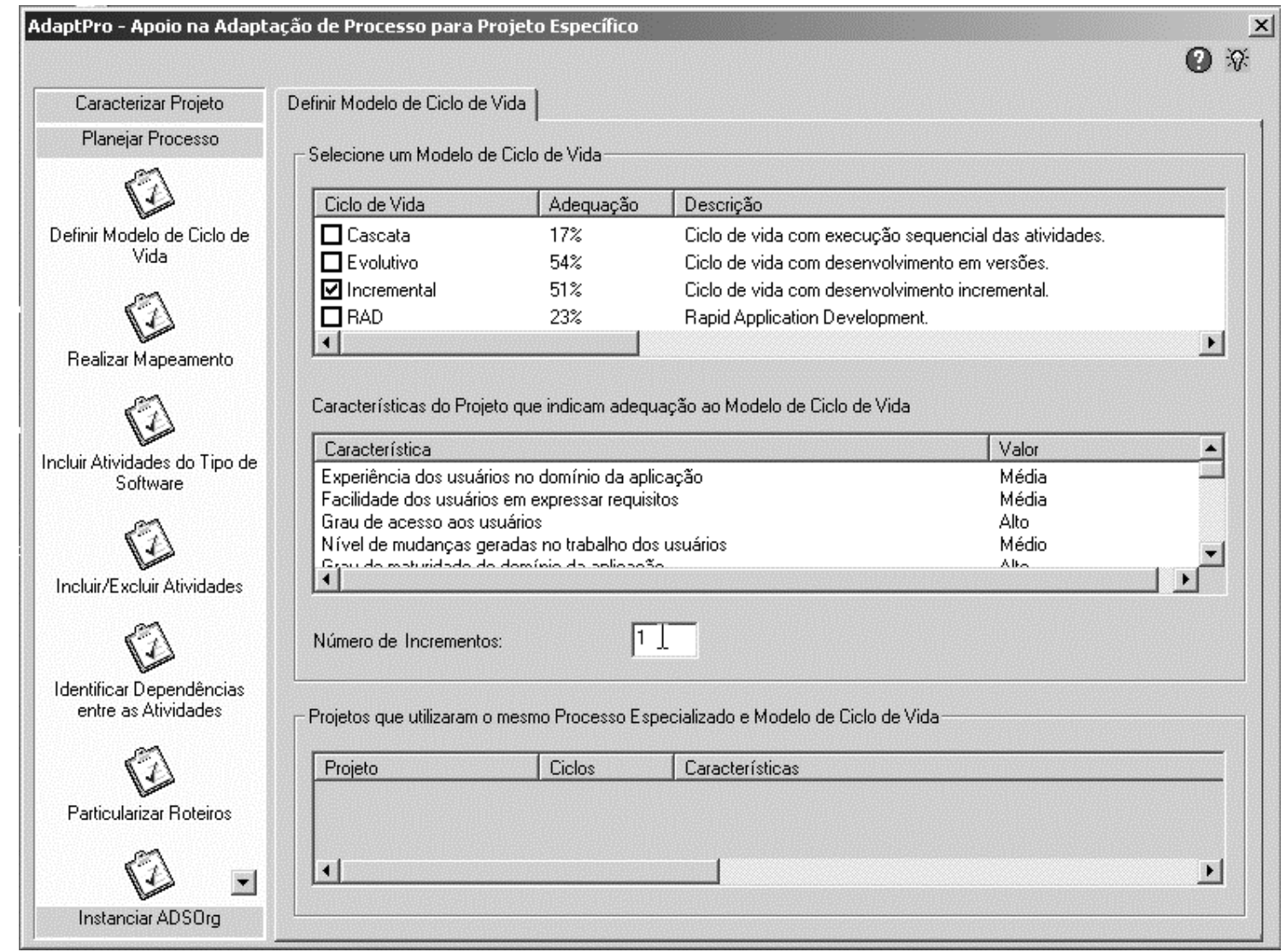

Figura 1 - AdaptPro - ferramenta de apoio a adapatação de processo de software

No lado esquerdo da Figura 1, o sistema apresenta as atividades que irão guiar a execução da ferramenta. No lado direito da figura, o sistema apresenta outra tela para apoiar a execução da atividade selecionada; neste caso, é apresentada a tela que apóia a 
definição de um modelo de ciclo de vida para um projeto específico como parte da atividade de planejamento do processo. Uma lista com os modelos de ciclo de vida e o nível respectivo de adequação para o projeto considerando suas características são apresentadas no lado direito da tela. Além do mais, o usuário pode consultar a justificativa da identificação automática do nível de adequação além dos processos de software definidos para projetos similares que utilizaram o mesmo ciclo de vida selecionado, facilitando a seleção de um modelo de ciclo de vida adequado pelo usuário.

Após o planejamento do processo, o gerente do projeto pode utilizar a ferramenta $\mathcal{A}$ dapt Pro para instanciar um processo específico para o projeto baseado nas suas particularidades. O produto desta ferramenta é o plano do processo (incluindo adaptações de acordo com o modelo de ciclo de vida escolhido) e um ADS para apoiar a execução do processo planejado. Através do ADS instanciado, o gerente do projeto pode, por exemplo, executar ferramentas de apoio a execução de atividades do processo. Uma dessas ferramentas é a $\mathcal{R H \mathcal { H }}$ lan [Santos et al. 2004], uma ferramenta para apoiar o planejamento de recursos humanos. A Figura 2 apresenta a tela desta ferramenta, exibindo um exemplo de alocação de recursos humanos nos projetos de atividades. No lado esquerdo é possível visualizar todas as atividades para planejamento de recursos humanos em um projeto.

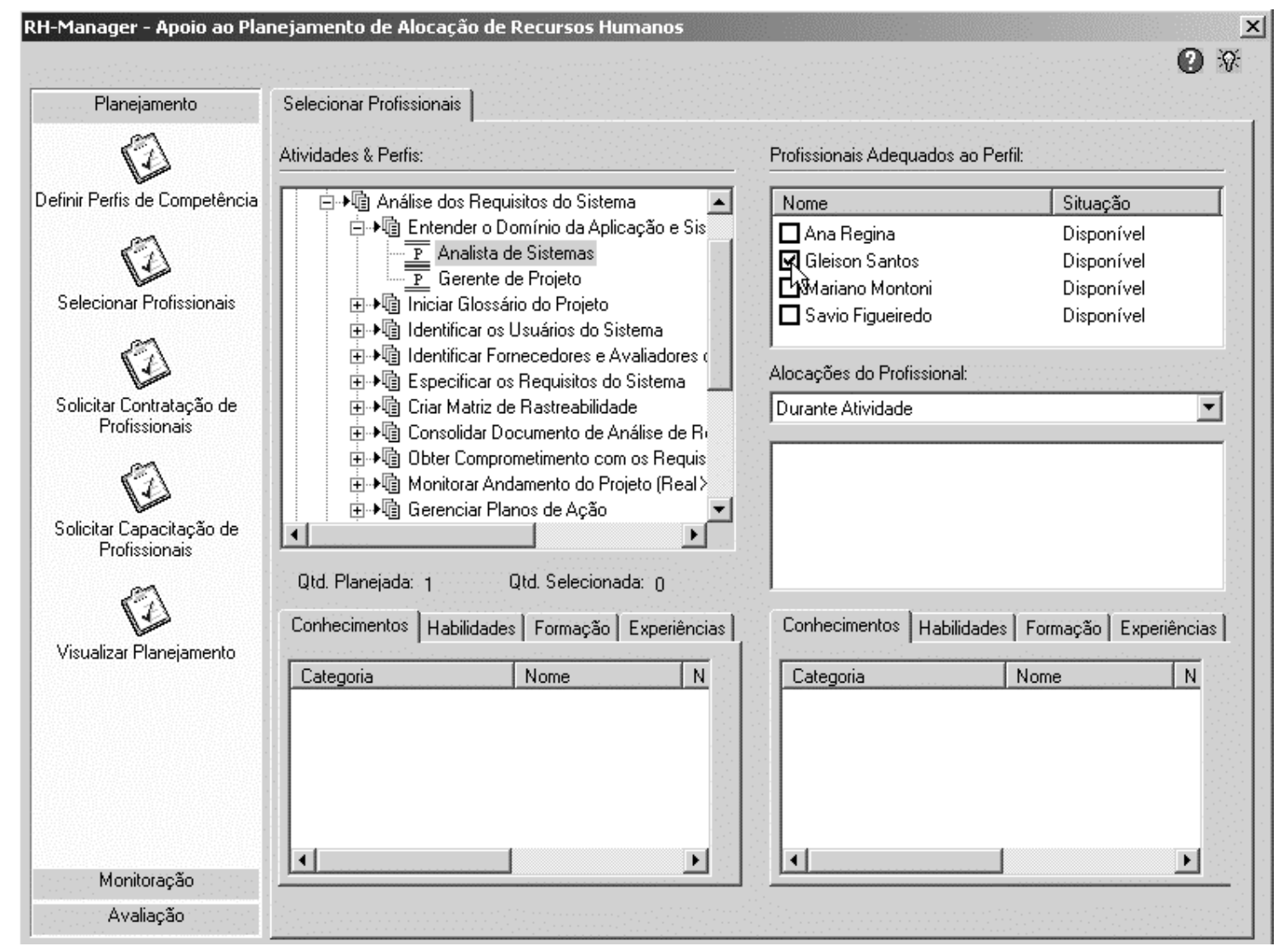

Figura 2 - Seleção de profissional na ferramenta RHPlan

O usuário pode também consultar as competências de cada usuário na ferramenta RHFPlan, além de poder consultar as análises de suas experiências passadas apoiando os 
gerentes de projeto no planejamento e alocação de recursos humanos de forma efetiva e eficiente. Experiências de projeto são uma importante fonte de conhecimento na Engenharia de Software e, portanto, as experiências adquiridas devem ser identificadas e compartilhadas. Além do mais, as lições aprendidas sobre alocações de recursos humanos em outros projetos são muito importantes para evitar que erros cometidos anteriormente não voltem a ocorrer.

Os benefícios obtidos pelo uso da Estação $\mathcal{T} a b a$ para apoiar a definição, desenvolvimento e melhoria de processos no contexto do Projeto mps $\mathrm{Br}$ são apresentadas na próxima seção.

\section{Resultados Práticos da Implantação do MR mps em Pequenas e Médias Empresas Brasileiras}

Os processos implantados em pequenas e médias empresas demonstraram diversos resultados, por exemplo, aumento da qualidade dos produtos e processos e preservação do conhecimento organizacional relacionado a processos de software. Um benefício direto obtido a partir dos processos implantados pode ser exemplificado através de duas empresas que obtiveram certificação ISO 9001:2000 baseado nos processos de software implantados.

A primeira empresa a obter a certificação ISO é uma empresa de desenvolvimento de software que durante dois anos esteve envolvida na definição, preparação e implantação de processos de software sem obter sucesso. Após um ano do início do projeto mps Br, a empresa obteve o certificado ISO 9000:2000.

A segunda empresa a obter o certificado da ISO é uma empresa de desenvolvimento de software que já possuía a certificação ISO 9000:1994 e tinha que estar aderente aos novos padrões da ISO 9000:2000 para manter a sua certificação. Segundo engenheiros de software da empresa, os processos implantados e o apoio da $\mathcal{E}$ s $a c ̧ a \tilde{a} o \mathcal{T} a b a$ foram decisivos para obter a renovação do certificado, porque aceleraram a implantação dos processos e facilitaram a disseminação de melhores práticas de desenvolvimento de software no nível organizacional.

Avaliações oficiais do MR mps serão executadas com o objetivo de avaliar uma empresa durante o primeiro semestre de 2005, e outras cinco empresas até o final deste ano. Uma dessas empresas também terá uma avaliação oficial do CMMI até o final de 2005 .

\subsection{Resultados Experimentais da Avaliação}

Um survey foi planejado e executado com o objetivo de analisar os processos implantados e o apoio das ferramentas da Estação $\mathcal{T} a b a$, com respeito à avaliação da adequação sob o ponto de vista de gerentes de projetos, analistas de sistemas e desenvolvedores no contexto de engenheiros de software executando os processos implantados com o apoio das ferramentas da Es tação $\mathcal{T} a b a$.

O survey foi executado através da aplicação de questionários a 16 membros das empresas que participaram da fase inicial do Projeto mps Br. Estes membros tiveram que preencher um formulário contendo conjuntos diversos de questões. As questões foram dividas em quatro seções. A primeira seção continha questões específicas com relação à 


\section{Simpósio Brasileiro de Qualidade de Software}

experiência dos participantes. A segunda seção continha questões relacionadas à implantação dos processos. A terceira seção continha questões sobre o apoio das ferramentas da $\mathcal{E}$ s $a c ̧ a \tilde{a} o \mathcal{T} a b a$. Finalmente, a seção quatro continha questões relativas às atividades e aos procedimentos específicos das áreas de processo. A Figura 3 apresenta os resultados da execução desta avaliação.

A partir dos resultados apresentados na Figura 3, pode-se perceber que as atividades e os procedimentos específicos das áreas de processo foram adequados para a maioria dos participantes. Além do mais, mais de $90 \%$ dos participantes reconheceram que a Es tação $\mathcal{T} a b a$ reduziu significativamente o esforço da execução da maioria das atividades dos processos. Apesar de cerca de $85 \%$ dos participantes declararem haver uma sensibilização adequada nas empresas quanto a importância do uso dos processos, quase $65 \%$ dos participantes notaram resistência na implantação desses processos. Para tratar esta divergência, a alta gerência demonstrou forte apoio para a implantação dos processos e estimulou os participantes a desenvolver os projetos de acordo com o processo definido. Como resultado disto, pode-se observar na Figura 3 que a maioria dos projetos foi sempre desenvolvido de acordo com os processos definidos e os membros das equipes reconheceram que esses processos estavam adequados aos projetos.

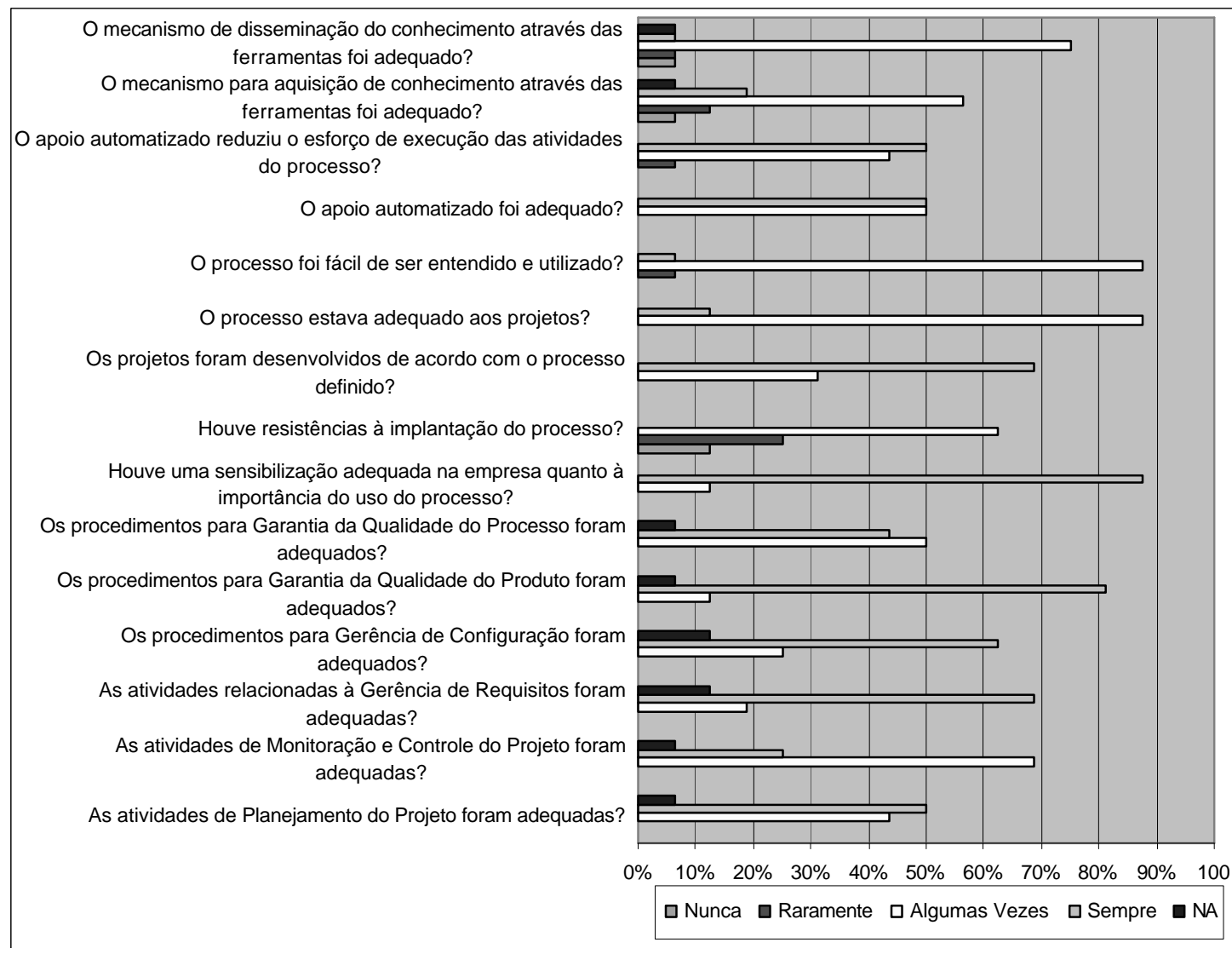

Figura 3 - Resultados da avaliação dos processos e do apoio da Est $a c ̧ a \tilde{a} o$ Ta $a$ a

Os participantes do survey também identificaram que tanto os processos quanto a Es tação Taba facilitaram a disseminação de melhores práticas desde as atividades de planejamento de projeto até a análise post mortem. Além do mais, a centralização de 
informações e conhecimentos relacionados à execução dos processos também apoiaram situações de tomada de decisão, pois os gerentes de projeto podiam facilmente consultar informações sobre projetos similares. A institucionalização dos processos com a Es tação Taba também facilitou a comunicação entre os membros dos projetos e diminuiu a ocorrência de desentendimentos ao longo do projeto com relação aos procedimentos e atividades a serem executados e os artefatos a serem produzidos.

\section{Lições Aprendidas}

Algumas lições foram aprendidas a partir dos resultados das experiências de implantação do MR mps com o uso da Estação TABA:

(i) um modelo genérico e abrangente é muito importante para permitir uma maior visibilidade dos processos implantados que dependem das particularidades e tamanho das empresas envolvidas;

(ii) a implantação cooperativa em um grupo de empresas tem se demonstrada adequada e capaz de satisfazer a realidade de pequenas e médias empresas, pois permite a implantação de um modelo com custos viáveis e mantendo a boa qualidade;

(iii) a experiência e conhecimento dos consultores e a existência de uma coordenação de um grupo de empresas que direciona as ações do grupo de forma adequada são aspectos fundamentais para o sucesso da implantação do MR mps;

(iv) o trabalho com um grupo de empresas demanda um alto número de consultores para prestar a atenção devida no momento em que a empresa mais necessita;

(v) a participação em um grupo demonstrou não ser adequada para empresas com um alto nível de especificidade ou que já possuem processos definidos e institucionalizados; nestes casos, implantação individual é mais adequada;

(vi) as atividades de treinamento para desenvolvedores, analistas de sistema e gerentes de projeto têm sido avaliadas positivamente; e

(vii) o apoio das ferramentas da Estação Taba demonstrou ser adequado para facilitar a implantação e utilização dos processos e para reduzir o tempo e esforço de institucionalização desses processos dentro da empresa.

\section{Considerações Finais}

Este trabalho apresentou a infra-estrutura da Estação TABA para apoiar a implantação do Modelo de Referência de Melhoria de Processo de Software Brasileiro. Também foram apresentadas a experiência piloto com relação à implantação da abordagem apresentada em empresas de software e as principais funcionalidades da Estação Taba.

Os resultados práticos da implantação do MR mps em pequenas e médias empresas e os resultados iniciais foram apresentados e analisados com o objetivo de identificar os benefícios da abordagem apresentada, tais como aumento da qualidade dos processos e produtos e preservação do conhecimento organizacional relacionado a processos de software. 
Um benefício direto do uso da Estação TABA pode ser exemplificado por três organizações que obtiveram a certificação ISO 9001:2000 [Ferreira et al. 2005] e uma empresa que foi avaliada com sucesso CMMI Nível 2 [Silva et al. 2005].

\section{Agradecimentos}

Os autores agradecem a todos os participantes do Projeto $\mathrm{mps} \mathrm{Br}$, os profissionais e empresas que participaram nesta experiência no Rio de Janeiro, e especialmente a Kival Weber e Eratóstenes Araújo, coordenadores do Projeto mps Br, a Benito Diaz Paret, coordenador da Riosoft, a Márcio Pecegueiro Amaral, coordenador do projeto Qualisoft.

\section{Referências}

Basili, V., Caldiera, G., Rombach, D., (1994), "The Goal Question Metric Paradigm", in Encyclopedia of Software Engineering, vol. 2: John Wiley and Sons, Inc.

Chrissis, M. B., Konrad, M, Shrum, S., (2003), "CMMI: Guidelines for Process Integration and Product Improvement", Addison-Wesley.

Farias, L., Travassos, G. H., Rocha, A. R. C., (2003), "Knowledge Management of Software Risks", In: Journal of Universal Computer Science, Vol. 9, No 7, 670- 681.

Ferreira, A. I. F., Cerqueira, R., Rocha, A. R., Santos, G., Montoni, M., Mafra, S., Figueiredo, S., (2005), "Implantação de Processo de Software na BL Informática Um Caso de Sucesso", IV SBQS, Porto Alegre, Brasil.

ISO 9001, (2000), "Quality management systems - Requirements.

ISO/IEC 12207, (2000), "Information technology -software process life cycle".

ISO/IEC 15504, (2003), "Information Technology - Process Assessment, - Part 1: Concepts and Vocabulary".

MCT (Ministério da Ciência e Tecnologia), SEPIN (Secretaria de Política de Informática), Qualidade e Produtividade no Setor de Software - Resultados da Pesquisa, 2002. http://www.mct.gov.br/sepin/dsi/quali2001/public2001.htm

Montoni, M., Miranda, R., Rocha, A. R., Travassos, G. H., (2004a), "Knowledge Acquisition and Communities of Practice: an Approach to Convert Individual Knowledge into Multi-Organizational Knowledge", In: Lecture Notes in Computer Science (LNCS), ISBN 3-540-22192-1, 6th Int. Workshop on Learning Software Organizations (LSO'2004), Banff, Canada, Jun. 110-121.

Montoni, M., Santos, G., Villela, K., Miranda, R., Rocha, A.R., Travassos, G.H., Figueiredo, S., Mafra, S., (2004b), "Knowledge Management in an EnterpriseOriented Software Development Environment", In: Proc. of the 5th Int. Conf of Practical Aspects of Knowledge Management, Vienna, Austria, 117-128.

Oliveira, K, Zlot, F., Rocha, A. R., Travassos, G., Galotta, C., Menezes, C. Domain "Oriented Software Development Environment, Journal of Systems and Software", vol 72/2 (2004) pp 145-161.

Santos, G., Villela, K., Schnaider, L., Rocha, A. R., Travassos, G. H., (2004), "Building Ontology Based Tools for a Software Development Environment”, In: Lecture Notes 
in Computer Science (LNCS), ISBN 3-540-22192-1, 6th Int. Workshop on Learning Software Organizations (LSO'2004), Banff, Canada, Jun. 19-30

Silva, R. P., Nunes, E. D., Rocha, A. R., Natali, A. C., Santos, G., (2005), "Uma Abordagem para Implantação de Processos de Software com ISO 9001 e CMMI", IV Simpósio Brasilieiro de Qualidade de Software, Porto Alegre, Brasil.

Villela, K., Santos, G., Montoni, M., Berger, P., Figueiredo, S, M., Mafra, S, N., Rocha, A, R, C., Travassos, G, H., 2004, "Definição de Processos em Ambientes de Desenvolvimento de Software Orientados a Organização", III Simpósio Brasileiro de Qualidade de Software, Brasília-DF.

Veloso, F., Botelho, A. J. J., Tschang, T., Amsden, A., (2003), "Slicing the Knowledgebased Economy in Brazil, China and India: A Tale of 3 Software Industries", In.: Report. Massachusetts Institute of Technology (MIT), Sep.

Weber, K. C., Pinheiro, M.: Software Quality in Brazil, In.: Quality World Magazine, The Institute of Quality Assurance (IQA), London, UK, Vol. 21, Issue 1.1, Nov. (1995)

Weber, K. C., Almeida, R.A.R., Amaral, H.G., Gunther, P. S., Xavier, J.H.F., Loures, R., (1997), "ISO 9001/TickIT Certification in Brazilian Software Companies", In.: 5th Int. Conf. on Software Quality Management (SQM'97), Bath, UK, Mar.

Weber, K. C., Rocha, A. R. C., Nascimento, C. J.: Qualidade e Produtividade em Software, 4a edição renovada. São Paulo, Makron Books, (2001)

Weber, C. K, Rocha, A. R; Alves, A, Ayala, A. M, Gonçalves, A, Paret, B, Salviano, C; Machado, C. F, Scalet, D, Petit, D, Araújo, E, Barroso, M. G, Oliveira, K, Oliveira, L. C. A, Amaral, M. P, Campelo, R. E. C; Maciel, T. , (2004), "Modelo de Referência para Melhoria de Processo de Software: uma abordagem brasileira", XXX Conferencia Latino-americana de Informática, Arequipa, Peru.

Weber, C. K., Machado, C. A. F., Scalet, D., Salviano, C. F., Rocha, A. R., (2005), "Modelo de Referência e Método de Avaliação para melhoria do processo de software - versão 1.0 (MR mps e MA mps)", IV Simpósio Brasilieiro de Qualidade de Software, Porto Alegre, Brasil. 